\title{
Muséologies
}

Les cahiers d'études supérieures

muséologies

\section{Le mentefact au musée : la mémoire mise en scène}

\section{Ariane Blanchet-Robitaille}

Volume 6, numéro 1, 2012

URI : https://id.erudit.org/iderudit/1011532ar

DOI : https://doi.org/10.7202/1011532ar

Aller au sommaire du numéro

\section{Éditeur(s)}

Association Québécoise de Promotion des Recherches Étudiantes en Muséologie (AQPREM)

ISSN

1718-5181 (imprimé)

1929-7815 (numérique)

Découvrir la revue

Citer cet article

Blanchet-Robitaille, A. (2012). Le mentefact au musée : la mémoire mise en scène. Muséologies, 6(1), 55-75. https://doi.org/10.7202/1011532ar

\section{Résumé de l'article}

Considérant la préoccupation grandissante qui existe quant au lien entre l'individu et son patrimoine immatériel en nouvelle muséologie, Ariane Blanchet-Robitaille précise la notion de mentefact et révèle comment l'intégration progressive de ce nouvel « objet muséal » au sein des collections énonce le rapport qui existe entre la mémoire et le musée. Envisageant les possibilités multiples qu'instaure cette nouvelle réalité pour le musée, dont la mise en valeur des contenus d'exposition, l'auteure souligne la nécessité pour l'institution muséale de se doter d'outils visant à faciliter l'acquisition, la documentation et la conservation des mentefacts. S'inspirant à la fois de la typologie du patrimoine immatériel élaborée par l'UNESCO et de la Grille des pratiques culturelles de Jean Du Berger qui classifie le patrimoine ethnologique, elle propose une typologie des mentefacts déclinée en cinq catégories, ainsi qu'une grille de sélection visant à encadrer leur intégration au sein des collections muséales. Son article fait également état de certaines mises en garde associées à l'intégration au musée des mentefacts, notamment en ce qui concerne le fait qu'ils doivent permettre la production d'un discours qui reflète la diversité caractéristique de notre réalité et qu'ils doivent aussi permettre de révéler le changement et le dynamisme inhérents à toute société, à travers le temps.
Tous droits réservés (C) Association Québécoise de Promotion des Recherches Étudiantes en Muséologie (AQPREM), 2012
Ce document est protégé par la loi sur le droit d'auteur. L'utilisation des services d'Érudit (y compris la reproduction) est assujettie à sa politique d'utilisation que vous pouvez consulter en ligne.

https://apropos.erudit.org/fr/usagers/politique-dutilisation/ 
Article trois

\section{Le mentefact au musée:}

\section{la mémoire mise en scène}

Ariane Blanchet-Robitaille 
Considérant la préoccupation grandissante qui existe quant au lien entre l'individu et son patrimoine immatériel en nouvelle muséologie, Ariane Blanchet-Robitaille précise la notion de mentefact et révèle comment l'intégration progressive de ce nouvel "objet muséal" au sein des collections énonce le rapport qui existe entre la mémoire et le musée. Envisageant les possibilités multiples qu'instaure cette nouvelle réalité pour le musée, dont la mise en valeur des contenus d'exposition, l'auteure souligne la nécessité pour l'institution muséale de se doter d'outils visant à faciliter l'acquisition, la documentation et la conservation des mentefacts. S'inspirant à la fois de la typologie du patrimoine immatériel élaborée par l'UNESCO et de la Grille des pratiques culturelles de Jean Du Berger qui classifie le patrimoine ethnologique, elle propose une typologie des mentefacts déclinée en cinq catégories, ainsi qu'une grille de sélection visant à encadrer leur intégration au sein des collections muséales. Son article fait également état de certaines mises en garde associées à l'intégration au musée des mentefacts, notamment en ce qui concerne le fait qu'ils doivent permettre la production d'un discours qui reflète la diversité caractéristique de notre réalité et qu'ils doivent aussi permettre de révéler le changement et le dynamisme inhérents à toute société, à travers le temps.

\footnotetext{
Ariane Blanchet-Robitaille est titulaire d'un baccalauréat intégré en sciences historiques et études patrimoniales ainsi que d'un diplôme d'études supérieures spécialisées en muséologie de l'Université Laval. Après l'obtention de son diplôme de deuxième cycle en 2010, elle a collaboré à différents projets d'exposition. Elle s'intéresse notamment aux questions liées à la recherche et à la diffusion ainsi qu'à l'intégration du patrimoine immatériel au musée. ariane.blanchet-robitaille.1@ulaval.ca
} 
Le musée, depuis quelques décennies, fait face à une préoccupation grandissante pour l'individu et ses productions immatérielles. Dans la vague de la nouvelle muséologie et des théories de Georges Henri Rivière' ${ }^{1}$, d'André Desvallées $^{2}$ et de Duncan Cameron ${ }^{3}$, de nouvelles façons de penser la relation entre le musée et son public ont été développées. Du «musée temple» au «musée forum », l'institution muséale s'est progressivement tournée vers la société et la communauté en adoptant des missions et des fonctions inédites. De nouvelles pratiques muséologiques ont vu le jour, telles que l'écomuséologie, la muséologie participative et la muséologie sociale. Issus de ces réflexions, le musée de société et les institutions qui en relèvent ont investi de nouveaux champs de pratique. Par ailleurs, le musée s'est progressivement dématérialisé, par l'apport des progrès technologiques, offrant de nouvelles possibilités à l'expographie, mais également en raison de l'importance croissante du patrimoine immatériel dans la construction et la valorisation identitaire des groupes culturels. Le musée est donc devenu ce lieu où l'identité et la mémoire collective sont recueillies et mises à la vue du public. Isac Chiva préfère d'ailleurs regrouper ces institutions sous la nomination de "musées d'identités collectives ", qui permet d'exprimer ce lien intrinsèque entre la nouvelle muséologie et la communautét ${ }^{4}$ En outre, le musée est désormais fréquenté pour assouvir un besoin d'insertion culturelle ou sociale et d'enracinement ${ }^{5}$. Du coup, le mouvement de la nouvelle muséologie et les pratiques qui s'en sont inspirées l'ont bien démontré: l'objet matériel, à lui seul, ne suffit plus à la représentation complexe de la société et de son histoire. Gaynor Kavanagh explique le phénomène en ces termes:

1 RIVIÈRE, Georges Henri. La muséologie selon Georges Henri Rivière: cours de muséologie, textes et témoignages. Paris: Dunod, 1989.

2 DESVALLÉES, André (dir.). Vagues: une anthologie de la nouvelle muséologie. Mâcon: Éditions W, 1992, 2 tomes. 3 CAMERON, Duncan. "The Museum: A Temple or the Forum ". Journal of World History. vol. 14, n ${ }^{\circ}$ 1, p. 189-202. 4 Conférence d'Isac Chiva présentée lors du colloque national Musées et Société à Mulhouse en 1991. Citée dans MAIROT, Philippe. "Musées et société». Ethnologie française, vol. XXVI, n 3, 1997, p. 346.
Are museums about object or people? [...] Traditionally, museums were places which housed and exhibited selected objects; curators tended the inanimate. But once the emphasis shifted in the postwar years from antiquities to social, cultural and industrial histories, understanding the recent past through objects alone became impossible to sustain or indeed justify. As soon as museums moved from antiquities to histories, they were committed to embracing a wide range of source materials.

Dans cette perspective, les musées de société et d'histoire ont investi de nouveaux terrains de recherche et de pratique, en mettant à contribution les études sur l'individu et ses manifestations relevant des sciences humaines et sociales. Dès lors, l'institution muséale s'est enquise de ces savoirs dans l'optique d'intégrer à ses activités de nouveaux objets, ceux-ci immatériels ${ }^{7}$. Ces nouveaux objets, qui bénéficient à la fois des avancées technologiques et de l'engouement pour le patrimoine immatériel, sont ici définis sous le terme de mentefact. Malgré la popularité indéniable du patrimoine immatériel au sein des institutions muséales, le manque de définition et d'outils empêche la nécessaire transition vers son application et son intégration cohérente dans les activités de conservation et de mise en valeur. Une nécessité émerge alors pour le développement de certaines procédures encadrant cette transition du patrimoine immatériel, d'une forme évolutive à une forme stable. Quelle conception le musée doit-il se faire du mentefact et quels sont les outils qui permettraient son intégration au sein de ses activités?

5 MAIROT, Philippe. "Identités et musées de société". In. LE GOFF, Jacques (dir.). Patrimoine et passions identitaires : Entretiens du patrimoine. Paris : Fayard, Éditions du patrimoine, 1998, p. 168.

6 KAVANAGH, Gaynor. Dream Spaces: Memory and the Museum. London: Leicester University Press, 2000, p. 7. 7 La conférence générale du Conseil international des musées (ICOM) de 2004, tenue sous le thème de "Musées et patrimoine immatériel ", témoigne de cette préoccupation pour le patrimoine vivant au sein des institutions muséales. 


\section{Définition de mentefact}

Le concept de mentefact est compris de différentes façons par les organismes et les auteurs qui en font l'usage. Bien que la littérature sur le sujet demeure très limitée, il est toutefois nécessaire de faire un survol des définitions existantes afin d'établir une interprétation du mentefact cohérente avec son intégration au musée.

Tel qu'énoncé par le ministère de la Culture, des Communications et de la Condition féminine (MCCCf), le mentefact représente l'« ensemble des productions spirituelles ou intangibles qui constitue le volet immatériel du patrimoine ethnologique $»^{8}$. Cette définition permet de regrouper sous le même concept l'ensemble des manifestations immatérielles produites par l'homme et représentant une part considérable du patrimoine culturel. Dans son État des lieux du patrimoine, des institutions muséales et des archives, l'Observatoire de la culture et des communications du Québec adopte un point de vue similaire en faisant référence au mentefact comme objet du patrimoine immatériel comprenant " les savoirs et les savoir-faire caractéristiques d'une collectivité, tels le patrimoine linguistique, la toponymie, le patrimoine scientifique, le patrimoine audiovisuel et le patrimoine d'expression $"{ }^{9}$. La tension dualiste entre le patrimoine matériel et le patrimoine immatériel est évoquée par Marie-Claude Rocher dans une étude sur le patrimoine des protestants francophones du Québec. Celle-ci explique:

[L]a typologie du patrimoine se conçoit généralement soit par relation au territoire (rural/urbain, local/mondial, mobilier/ immobilier), soit par une relation au présent (formes " vivantes "/formes " mortes ",

8 Mentionnons que cette définition est également partagée par la Politique du patrimoine de la République française. (MINISTËRE DE LA CULTURE, DES COMMUNICATIONS ET DE LA CONDITION FÉMININE. Glossaire. <http://www.mcccf.gouv.qc.ca/ index.php?id=1899> [consulté le 30 janvier 2010].) 9 OBSERVATOIRE DE LA CULTURE ET DES COMMUNICATIONS DU QUÉBEC (OCCQ). État des lieux du patrimoine, des institutions muséales et des archives. Cahier 1: Premier regard. <http://www.stat.gouv.qc.ca/ observatoire/publicat_obs/pdf/cahier1etat_patrimoine.pdf> (consulté le 20 mars 2010). historique/contemporain), soit par fonction ou discipline (religieux, architectural, industriel, naturel, résidentiel...), soit encore par des variations du binôme concret/abstrait (tangible/intangible, mentefacts/artefacts, matériel/immatériel $)^{10}$.

Ces définitions permettent d'amorcer la réflexion sur le mentefact en l'introduisant dans le champ du patrimoine immatériel, tel que l'ethnologie le conçoit, sans nécessairement faire allusion à sa portée muséale.

Certains auteurs définissent le mentefact en lien avec le domaine muséal. Par exemple, selon Jan Dolák, le mentefact s'inscrit dans une classification de la collection qui se distingue ainsi: « it can be an artefact (created by Man), a "naturfact" (created by nature) or a "mentefact" (a recording of the intangible manifestations of man), subject in all cases to strict selection criteria ${ }^{11}$. En outre, d'après cet auteur, il va de soi que le mentefact est approprié dans les activités du musée, du moins parmi celles relevant de ses collections. Par le fait même, le mentefact n'a plus à être considéré comme étant uniquement un savoir-faire qu'il est possible de définir et d'observer, mais plutôt comme étant la trace de cette pratique que le musée se doit de conserver. Dans les termes muséologiques de base, publiés dans la revue internationale de muséologie Publics et musées, le mentefact est également défini comme objet muséal:

Parallèlement aux artefacts, il existe une autre catégorie d'objets fabriqués par l'homme que l'on désigne généralement par le terme de mentefacts. Alors que les artefacts et les naturalia correspondent à des choses concrètes, que l'on perçoit, le terme mentefact renvoie à des données abstraites, indépendamment de leur support physique ${ }^{12}$.

10 ROCHER, Marie-Claude. "Double traîtrise ou double appartenance?: Le patrimoine des protestants francophones au Québec”. Ethnologies, vol. 25, nº 2, 2003, p. 221.

11 DOLÁK, Jan. «Museology as a Tool for a Modern Museum ». In Actes du colloque Tourisme culturel: tendances et stratégies. <http://www.montrealceca2008.org/12.html> (consulté le 27 mars 2010).

12 "Termes muséologiques de base: Objet". Publics et musées: revue internationale de muséologie, $\mathrm{n}^{\circ}$ 14, 1998, p. 167. 
Cette volonté de différencier les productions matérielles et immatérielles de l'homme anime plus particulièrement la réflexion sur l'objet muséal. Dans ce même cadre de réflexion, Jean Du Berger a réfléchi sur la question de l'oralité au musée et il fait mention de la transmission des savoirs et des pratiques en tant que mentefact. Celui-ci souligne au sujet des pratiques culturelles: "dans ce mode de transmission, les objets de transmission ne sont pas conservés sous une forme stable: il s'agit d'"un-recorded mentifact' " ${ }^{13}$. À cette étape du processus de transmission, les savoir-faire ne sont pas enregistrés ni inscrits sous une forme stable qui en permettrait la conservation et la diffusion. Le mentefact ne peut être, à ce moment, considéré comme objet muséal. Dans cette perspective, le passage de ce "un-recorded mentifact", relevant de la transmission des pratiques culturelles, au mentefact, "objet de musée», est intimement lié aux progrès techniques des derniers siècles. Selon Mariannick Jadé:

L'atout des nouvelles technologies élargit les capacités de conservation de toutes formes de réalités. Elles inscrivent le fugace, le captent dans toute sa complexité et assurent la capitalisation d'une connaissance complète du réel tel qu'il a existé dans toute son authenticité. Les duplications numériques respectent avec justesse son aspect formel (chansons, situations, films d'expériences...) en saisissant sa nature expressive sonore ou visuelle, bien plus juste que celle de l'écrit ${ }^{14}$.

Ainsi, l'enregistrement permet de saisir la complexité d'une performance en reproduisant une pluralité de dimensions sensorielles, imperceptibles par l'écrit et même par l'image. En ce sens, le mentefact apporte de nouvelles possibilités aux activités muséales par la portée de son contenu et son caractère multidimensionnel. Jadé poursuit en affirmant:

Les substituts sont aujourd'hui très variés et portent sur des événements du passé très précis. Duncan Cameron et André Desvallées les ont répertoriés: ce sont

13 DU BERGER, Jean. "Prolégomènes à une ethnologie urbaine ". In. TURGEON, Laurier, Jocelyn LÉTOURNEAU et Khadiyatoulah FALL (dir.). Les espaces de l'identité. Québec: Presses de l'Université Laval, 1997, p. 213. des kinefacts, des mentefacts... Captés sur supports de conservation, ces traces doivent être qualifiées par l'adjectif "intangible" car ils sont fixés dans le temps de la saisie documentaire. Le support-témoin doit ainsi être considéré comme une preuve matérielle de ce qui a existé mais qui a disparu ${ }^{15}$.

Dans cette optique, le mentefact est compris comme la trace d'un événement, d'une performance ou d'un entretien ayant eu lieu, mais qui n'est plus. Le mentefact constitue alors le témoin de ce qui s'est produit, un re-création de l'événement sans être l'événement lui-même, un "substitut", tel que l'observe Jadé. Il nécessite un support matériel (bande magnétique, $\mathrm{CD}, \mathrm{DVD}$, etc.) qui assure sa conservation et surtout sa diffusion. Sans support adéquat, le mentefact ne peut donc être représenté dans toute sa complexité, d'où l'apport indéniable des progrès techniques et technologiques. Cette définition, proposée par Jadé, comporte plusieurs éléments intéressants qui permettent de définir le concept de mentefact tel que nous l'entendons. En somme, le mentefact relève du champ de l'abstrait et de l'immatériel et comprend l'ensemble des manifestations spirituelles ou intangibles de l'Homme. Dans ce cadre, une prière et un témoignage sont tous deux considérés comme des pratiques culturelles, d'où la question suivante: Est-ce que la prière ou le témoignage sont considérés en soi comme des mentefacts ou est-ce plutôt l'objet muséalisé, l'enregistrement de ces performances qui constitue le véritable mentefact? Nous adopterions plutôt la seconde position, puisque le mentefact est un substitut de l'événement, une re-création de ce dernier, comme le soutient Jadé.

Le support sur lequel est inscrit l'événement constitue l'aspect matériel par lequel le mentefact peut être conservé. Par ailleurs, alors que l'événement en soi est unique, le mentefact peut au contraire être diffusé de manière pratiquement illimitée, selon la stabilité de son support.

14 JADÉ, Mariannick. Le patrimoine immatériel: perspectives d'interprétation du concept de patrimoine. Paris: L'Harmattan, 2006, p. 58.

15 Id., p. 58. 
Ce dernier aspect constitue d'ailleurs l'une des raisons pour lesquelles il est désormais possible d'intégrer le mentefact au musée, tant au niveau des collections que des expositions. Inscrites sur support matériel, les manifestations du patrimoine immatériel sont conservables et diffusables, dans l'intégralité, ou presque, de leur complexité. Le mentefact représente en effet la trace se rapprochant le plus de l'événement lui-même, telle qu'une entrevue, un chant ou un conte, ce qui n'était autrefois possible que par des retranscriptions, des illustrations et, plus tard, des photographies. De ce fait, le mentefact fige la performance à son stade de réalisation et, par conséquent, dans un contexte de production révolu. Dans la réalité, ces performances continueront d'évoluer, au fil du temps et des contextes de production, ou ne seront tout simplement jamais reproduites. Le chant traditionnel enregistré subira probablement des modifications, majeures ou mineures, avec le temps et le contexte, ou même selon la personne qui l'interprétera. Il en va de même dans le cas d'une personne qui témoigne de sa vie. Cette même personne en fera un tout autre récit lorsque la même tâche lui sera demandée des années plus tard. Non seulement aurat-elle vécu de nouveaux événements, mais la sélection des épisodes de sa vie sera probablement différente. En somme, le mentefact représente la trace d'un événement unique, qui n'existe plus, mais qui peut être visionné de manière quasi illimitée. Enfin, rappelons que bien qu'il y soit intimement lié, le mentefact doit être considéré indépendamment du support sur lequel il est enregistré. Relevant du vaste champ des pratiques culturelles, il est donc indéniable que le mentefact puisse prendre diverses formes, notamment le conte, le chant, la performance artistique, le récit de vie, la danse, les rituels. Ces pratiques culturelles, investiguées par les chercheurs des sciences humaines et sociales, sont multiples, à l'image de la complexité de la société qu'elles prennent

16 FOULQUIÉ, Paul. Vocabulaire des sciences sociales. Paris: Presses universitaires de France, 1978, p. 350. 17 MESURE, Sylvie et Patrick SAVIDAND (dir.). Dictionnaire des sciences humaines. Paris: Presses universitaires de France, 2006, p. 557. pour objet d'étude. Le témoignage oral servira de cas d'analyse afin de restreindre l'étude à un type spécifique de mentefact.

\section{Le témoignage oral comme mentefact}

Le témoignage est considéré comme l'acte de rendre compte de ce qu'on a vu ou entendu; conséquemment, le témoin représente une "personne qui, ayant vu ou entendu certaines choses, en a une connaissance directe dont elle peut faire part oralement ou par écrit, en particulier dans une enquête judiciaire, mais aussi dans une enquête historique ou sociologique ${ }^{16}$. Le recours au témoin est depuis longtemps intégré aux pratiques des chercheurs en sciences humaines et sociales, qu'il s'agisse du sociologue ou de l'anthropologue qui utilisent le récit de vie comme matériau central de leurs recherches ou, plus récemment, de l'historien qui envisage le témoignage comme source orale ${ }^{17}$. Dans la même perspective, plusieurs institutions muséales incorporent désormais des témoignages oraux dans leurs expositions, puisque ceux-ci enrichissent la présentation tant par leur contenu documentaire que par leur nature dynamique. Intégrés à l'exposition, les témoignages permettent aux visiteurs d'entendre les voix de ceux qui ont utilisé les objets présentés ou de ceux qui ont vécu les événements décrits.

De multiples manifestations sont sous-jacentes à la notion de témoignage. Pour les comprendre, la typologie proposée par Gaynor Kavanagh dans un ouvrage s'intéressant au rapport entre la mémoire et le musée semble des plus pertinentes ${ }^{18}$. Cet auteur propose cinq catégories de témoignages oraux et les résume comme suit: les témoignages liés aux compétences, aux savoir-faire, aux pratiques et à l'usage des objets; les témoignages relevant des pratiques langagières; les témoignages liés à la mémoire épisodique, c'est-à-dire la mémoire relative à

18 KaVANAGH, Dream Spaces: Memory and the Museum, op. cit., p. 68. 
un ou plusieurs épisodes précis de la vie d'un individu; les témoignages reconstituant le récit de vie d'un individu; les témoignages liés à l'identité et aux croyances ${ }^{19}$. Ces cinq thèmes intégrateurs permettent de couvrir un vaste champ de manifestations.

Le témoignage oral mis en valeur au musée constitue un mentefact puisqu'il est substitut et re-création de l'entrevue au cours de laquelle il a été réalisé et qu'il est inscrit sur un support matériel. Kavanagh fait en outre mention de l'enregistrement de l'entrevue sur un support matériel et de l'immuabilité du discours que ce processus engendre:

The interview is tapping into one brief moment in an individual's life and through that moment is seeking to access information. But that same information is constructed out of the remembering self — at the specific instant — and may well change over time. The oral historian is attempting to arrest and formalize something which otherwise has fluidity and the capacity to change ${ }^{20}$.

Ainsi, le témoignage oral est intimement lié au travail de mémoire. Comme le constate Michel Bozon à la suite d'une étude réalisée auprès d'une communauté rurale affectée par l'urbanisation:

De l'identité de ce groupe social [...] il ne reste donc plus qu'une mémoire: mémoire des savoir-faire, des savoir-vivre, des savoir-dire, mémoire des lieux, mémoire de l'Histoire. Le seul patrimoine possédé par ce groupe social qui n'a aujourd'hui plus d'existence réelle est donc un patrimoine oral, une identité inscrite dans le passée ${ }^{21}$.

Cette mémoire est donc recueillie sous la forme de témoignages oraux, de mentefacts. Par ailleurs, le produit de l'entrevue constitue

19 Ibid.

20 Id., p. 86.

21 BOZON, Michel. «Mémoire et musée ». In. Séminaire

de l'École du Louvre. Quels musées, pour quelles fins aujourd'hui? Paris: Documentation française, 1983, p. 58.

22 DE GAULEJAC, Vincent et André LÉVY (dir.). Récits de vie et histoire sociale: quelle historicité. Paris: Éditions Eska, 2000, p. 38. une construction interactive ou «le regard d'un présent sur le passé [...] une mémoire qui produit du sens $"^{22}$. Le mentefact-témoignage, tout comme le musée lui-même, représente des constructions mémorielles subjectives et permet d'évoquer des contextes révolus ${ }^{23}$. C'est en ce sens précis que l'intégration du mentefact au musée constitue une mise en scène de la mémoire. La mémoire étant dynamique et évolutive, il est alors nécessaire de se questionner sur la valeur documentaire et scientifique d'un tel objet.

\section{Valeur documentaire et scientifique}

La valeur documentaire du témoignage semble la plus évidente, puisque ce dernier constitue d'abord et avant tout une source d'informations recueillies pour des besoins de recherche. En effet, «in terms of recording and collecting, memories enter the museum as product, as a source of information, something which can be stored, interpreted, returned to and employed ${ }^{24}$. Ainsi, le mentefact est intégré au musée en raison de son potentiel à être utilisé à des fins de recherche ou de diffusion. Si la valeur documentaire est inhérente au témoignage, sa valeur scientifique doit pour sa part être davantage justifiée. Selma Thomas avance que dans une exposition, les récits de vie, côtoyant des artefacts et des documents d'archives, doivent présenter la même validité historique et une crédibilité incontestable ${ }^{25}$. Par ailleurs, comme le mentefact relève systématiquement de la subjectivité de son créateur, surtout lorsque la mémoire de ce dernier est impliquée dans le processus, le récit qui en résultera sera construit selon une interprétation personnelle et influencée par le contexte dans lequel il est livré. Mariannick Jadé explique que:

23 RITCHIE, Donald A. Doing Oral History: A Practical Guide. Oxford: Oxford University Press, 2003, p. 237. 24 KAVANAGH, Dream Spaces: Memory and the Museum, op. cit., p. 3.

25 THOMAS, Selma. «Private Memory in a Public Space: Oral History and Museums ». In. HAMILTON Paula et Linda SHOPES (dir.). Oral History and Public Memories. Philadelphie: Temple University Press, 2008, p. 89. 
les hommes construisent leur mémoire. Ils ne font pas renaître les événements tels qu'ils ont existé car ils les interprètent. La re-convocation incessante des connaissances du passé pose la grande problématique du fait patrimonial: assurer l'équilibre entre l'objectif et le subjectif ${ }^{26}$.

Dans ce cadre, la quête muséale prendrait forme dans la constitution d'une juste mémoire, représentant l'équilibre entre les rapports objectif/ subjectif et oubli/souvenir. Néanmoins, les histoires de vie, ou plus exactement les récits d'histoires de vie, fondés sur des souvenirs, à la fois personnels et collectifs, non seulement ne recherchent pas l'objectivité, mais revendiquent au contraire le fait d'être conditionnés par un point de vue, marqué par des engagements particuliers ${ }^{27}$.

Le rapport dualiste objectivité/subjectivité ne peut donc pas être un déterminant de la valeur scientifique du mentefact. En tant que convocation de la mémoire, le témoignage est nécessairement subjectif, ce qui n'amenuise pas pour autant sa valeur scientifique. Mairot propose une réflexion des plus pertinentes à ce sujet, rappelant l'opposition traditionnelle entre la mémoire in vivo, fluide, dynamique et créative, à celle des musées et du patrimoine, in vitro, qui se veut pour sa part froide, injonctive et résistante. Mairot reprend les propos de Marc Guillaume dans son article «Invention et stratégies du patrimoine" :

Selon Marc Guillaume, si les deux mémoires ne se rencontrent pas, si la mémoire qu'il nomme symbolique ne digère pas à sa façon la mémoire qu'il nomme hétérotopi$q^{2} e^{28}$, pour produire «la fiction d'un passé commun », "la politique du patrimoine n'est qu'une forme vide ${ }^{29}$.

26 JADÉ, Le patrimoine immatériel: perspectives..., op.cit., p. 62

27 DE GAULEJAC et LÉVY, Récits de vie et histoire sociale..., op. cit, p. 53 .

28 Le concept d'hétérotopie a été développé par Michel Foucault lors d'une conférence intitulée "Des espaces autres" présentée en mars 1967 au Cercle d'études architecturales. Foucault proposait alors que, parallèlement aux utopies relevant de l'imaginaire pur, les hétérotopies constituent des formes d'utopies réalisées, ancrées dans le réel.
Par conséquent, le mentefact-témoignage, bien que de nature subjective, permet d'équilibrer cette mémoire in vitro, longtemps considérée comme la seule méritant d'être étudiée et présentée par le musée. Dans un même ordre d'idées, la présence du chercheur influence le contenu du témoignage, dès lors orienté par l'intention de connaissance, cette visée scientifique étant à l'origine de la rencontre entre l'enquêteur et le témoin.

Du coup, il importe d'appliquer des mesures supplémentaires permettant le contrôle des informations. Ces mesures, bien connues des chercheurs en sciences humaines et sociales, sont ainsi exprimées : " appels à d'autres sources et critique de ces sources, croisement des différents témoignages, mise en corrélation des documents d'époque et des récits oraux $»^{30}$. La méthode de croisement des différents témoignages est abondamment citée dans les études portant sur le sujet. Cette méthode, utilisée dans la poursuite de travaux scientifiques, peut également s'appliquer au cadre muséal de l'intégration du témoignage. L'institution muséale se doit d'acquérir, de conserver et de diffuser un ensemble représentatif des témoins de l'histoire d'une société. Il devient donc essentiel de disposer d'une multiplicité de voix permettant la production d'un discours le plus près possible de la réalité. Dans son guide de collecte d'histoires de vie, Donald Ritchie écrit à ce propos :

By incorporating multiple voices, the exhibits teach the public that history may be interpreted in different ways. Rather than the authoritative, and sometimes condescending, single voice of the historian/curator lecturing the visitor on what it all means, the mélange of voices of participants and commentators can argue with each other to recreate the complexity of the past ${ }^{31}$.

29 GUILLAUME, Marc. "Invention et stratégies du patrimoine ». In. JEUDY, Henri Pierre (dir.). Patrimoine en folie. Paris: Éditions de la Maison des sciences de l'homme, 1990, cité dans MAIROT, «Musées et société ", op. cit., p. 353. 30 DE GAULEJAC et LÉVY, Récits de vie et histoire sociale..., op. cit., p. 39. 31 RITCHIE, Doing Oral History: A Practical Guide, op. cit., p. 240. 
Ainsi, que ce soit par l'apport d'autres témoignages ou d'autres objets et documents, le mentefact nécessite une mise en perspective pour en assurer la validité scientifique. En ce sens, les institutions muséales doivent favoriser une collecte permettant le croisement des données, en s'assurant d'avoir assez de témoins pour garantir la validité de l'information recueillie. C'est ainsi que certaines institutions québécoises se sont démarquées par leurs initiatives et constituent de véritables chefs de file en la matière. Tel est le cas notamment du Musée de la mémoire vivante de Saint-Jean-Port-Joli et du Centre d'histoire de Montréal.

\section{Le témoignage comme mentefact au musée:} deux études de cas

Inauguré en 2008, le Musée de la mémoire vivante (MMV) se consacre à la conservation et à la diffusion du patrimoine immatériel. Élaboré dès 2004 dans la vague de la Convention pour la sauvegarde du patrimoine immatériel de l'UNESCO, le concept du MMV est alors des plus novateurs au Québec.

Espace de création, d'hospitalité, de discussions, de fêtes et de direction, il se consacre aux histoires de vie et aux témoignages sous toutes leurs formes (orales, écrites, graphiques, audiovisuelles, numériques, etc.). Il conserve, étudie et met en valeur la mémoire de ses publics dans le but d'enrichir leur compréhension du monde et afin de transmettre ces repères culturels aux générations futures. Cette institution est en soi une mémoire vivante en constante évolution ${ }^{32}$.

Un tel énoncé de mission témoigne de la place centrale qu'occupe la source orale dans les activités de cette institution muséale. La mémoire étant fondamentale à la démarche mise de l'avant par le musée, ses collections

\section{MUSÉE DE LA MÉMOIRE VIVANTE.}

Mission. <http://www.memoirevivante.org/index.html> (consulté le 30 janvier 2010).

33 Fondé en 2007, le programme du Musée de la mémoire vivante à l'école permet à des jeunes du primaire et du secondaire de devenir enquêteurs d'un jour en rencontrant des personnes aînées et en enregistrant leurs témoignages. Par ailleurs, le Musée de la mémoire vivante sur la route sont essentiellement constituées d'enregistrements audio. Les témoignages recueillis et intégrés aux collections sont des plus variés, tant par la nature de leur contenu que par leur contexte de collecte. Pour la majorité, ils ont été collectés au sein même des locaux du musée ou lors de la tenue de programmes du Musée de la mémoire vivante sur la route et du Musée de la mémoire vivante à l'école, activités réalisées avec la collaboration de divers associations et établissements scolaires de la région ${ }^{33}$. Les expositions présentées par le MMV constituent un moyen privilégié de diffuser la richesse des documents oraux. En effet, l'objet matériel y agit en tant que déclencheur de mémoire plutôt qu'artefact central au propos, tandis que les extraits de témoignages diffusés occupent une place centrale dans la mise en valeur des thèmes. Dans cette optique, les expositions offrent aux visiteurs un espace de consultation qui leur permet de naviguer dans la base de données, véritable collection mémorielle, donnant ainsi accès à tous les documents enregistrés. Il s'agit ici d'une mémoire plurielle et inclusive, recueillie dans le but de valoriser l'histoire personnelle de chaque individu, considérant que chacune de ces histoires mérite une attention particulière.

Le Centre d'histoire de Montréal (CHM) et le Musée de la personne de Montréal (MPM) constituent également des exemples éloquents de l'intégration du mentefact au musée. Fondé en 1983 en tant que centre d'interprétation sur l'histoire de Montréal, le CHM favorise l'intégration des différentes communautés de la métropole par le développement de multiples programmes et activités à leur intention. Par ailleurs, le Musée de la personne de Montréal, fondé en 2004 à l'initiative du CHM, constitue un musée virtuel qui œuvre dans la collecte et la mise en valeur des histoires de vie.

permet au musée d'aller à la rencontre des gens à l'occasion de différents rassemblements (festivals, sociétés d'histoires, regroupements familiaux, etc.) et de recueillir de nombreux témoignages. Ces programmes ont été mis sur pied par l'institution muséale dans le but de se rendre accessible à un plus grand nombre de personnes. 


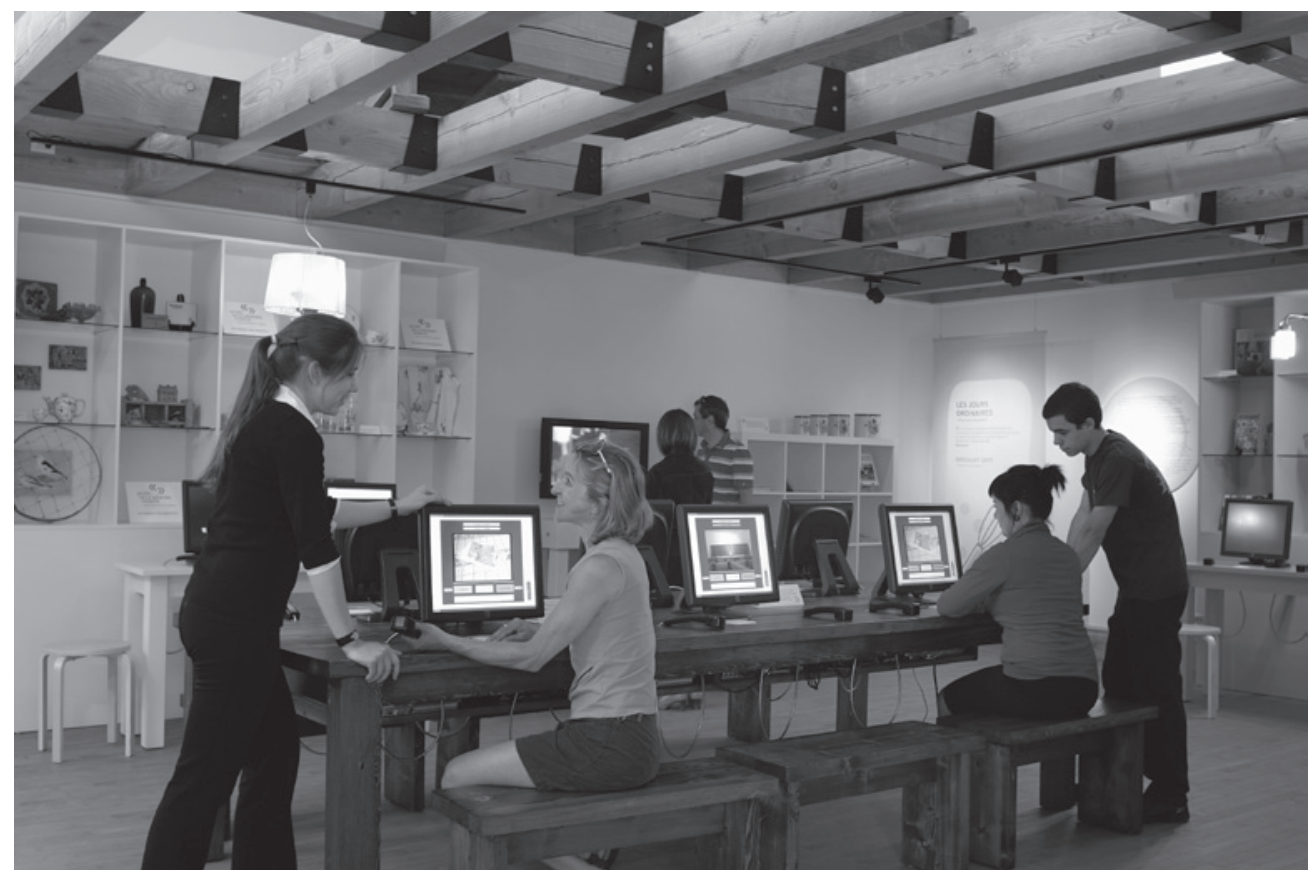

Illustration 1

Postes d'écoute dans l'exposition "Souvenirs de table", Musée de la mémoire vivante.

(c) Musée de la mémoire vivante [Photographe: Michel Julien] 


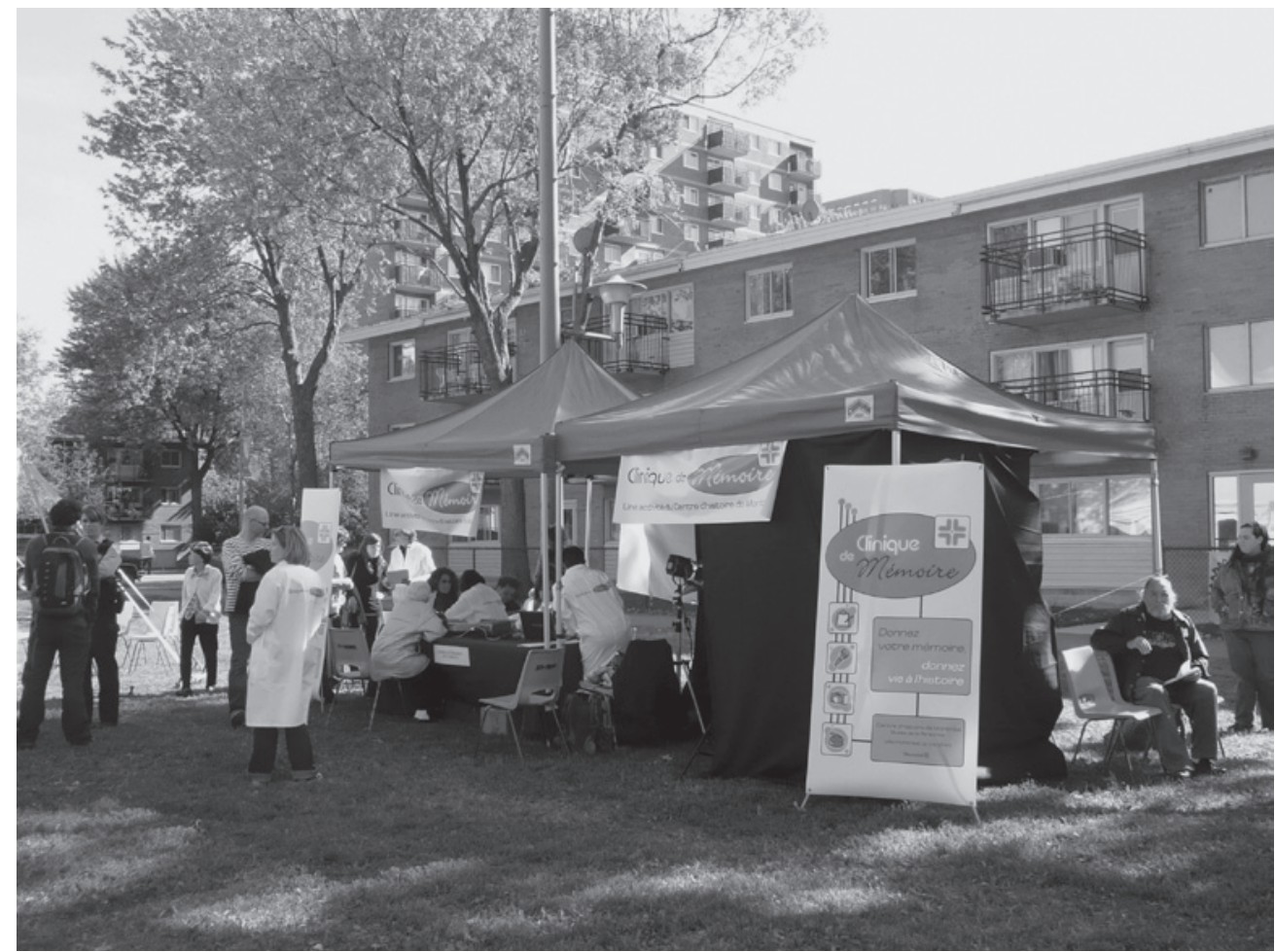

Illustration 2

Clinique de mémoire aux Habitations

Jeanne-Mance, Centre d'histoire de Montréal, Musée de la Personne de Montréal.

(C) Centre d'histoire de Montréal, Musée de la Personne

de Montréal [Photographe: Ariane Blanchet-Robitaille]. 
Héritière du réseau international des Musées de la personne, cette institution muséale a pour mission de permettre au plus grand nombre de personnes d'enregistrer, de préserver et de diffuser leur histoire de vie, suivant le principe que chaque mémoire individuelle mérite d'être sauvegardée. Les deux institutions muséales œuvrent conjointement au projet de clinique de mémoire, mis sur pied en 2003. Cette initiative, s'inspirant du modèle de la collecte de sang, se définit comme suit: La clinique de mémoire est une opération de collecte et de socialisation de la mémoire d'un groupe. Utilisant les méthodes d'histoire orale, dont l'entrevue filmée, elle se déroule sur une ou plusieurs journées. Elle vise à créer une collection de témoignages et à valoriser ce patrimoine, mais également à susciter, au moment de la collecte, une transmission directe de la mémoire entre les membres de la communauté ${ }^{34}$.

Ces cliniques de mémoire permettent de recueillir des témoignages pour leur éventuelle diffusion à l'occasion d'une exposition, réelle ou virtuelle, mais également dans l'objectif d'établir des liens avec les différentes communautés et entre elles. Ce concept de clinique de mémoire permet de réinventer les formes d'intervention dans le domaine patrimonial, notamment auprès des communautés culturelles. Depuis la toute première expérience, réalisée auprès de la communauté portugaise de Montréal, plusieurs cliniques de mémoire ont été organisées, notamment auprès des résidents des Habitations Jeanne-Mance et de ceux de l'arrondissement Saint-Laurent. Par ces activités de collecte, le Centre d'histoire de Montréal ainsi que le Musée de la personne de Montréal s'insèrent de manière probante dans la réflexion sur l'utilisation du mentefact au musée.

Le travail de ces deux institutions permet de constater la variété des témoignages qui peuvent être intégrés au musée, tant sur le plan des activités de diffusion que celui des collections. Au Musée de la mémoire vivante, le mentefact est au centre des préoccupations. Les témoignages constituent les objets de collection, à la fois recueillis, conservés et mis en valeur. Dans cette dynamique, les objets matériels n'agissent qu'en tant que supports visuels. Le Centre d'histoire de Montréal s'intéresse également à la conservation du patrimoine immatériel sans s'y consacrer entièrement. Quoique bien développé, il s'agit plutôt d'un secteur d'activité de l'institution, permettant de diffuser le patrimoine de communautés dont l'héritage matériel est incomplet. Les contextes d'utilisation évoqués traduisent également la diversité des statuts pouvant être octroyés au mentefact dans sa relation avec les autres objets et documents. La légitimité du patrimoine mémoriel n'est plus à discuter pour ces institutions, conscientes de la valeur inhérente de ces manifestations intangibles. Leurs activités de collectes mémorielles ainsi que les structures mises en place pour la documentation, la conservation et la diffusion des témoignages sont indéniablement adaptées à l'intégration du patrimoine immatériel au musée. Néanmoins, ces démarches s'inscrivent dans la volonté de préserver et de partager l'histoire de vie de tous ceux qui le désirent. Ce principe d'inclusion du plus grand nombre de témoignages pourrait éventuellement s'avérer problématique en raison de la charge de travail que cela pourrait engendrer si aucune balise n'est fixée. Ainsi, la mise en place d'outils permettant l'identification et la sélection des témoignages serait des plus pertinentes pour l'intégration du mentefact au musée.

\section{Vers une typologie du mentefact au musée}

Les institutions muséales, devant cet engouement pour le patrimoine immatériel, doivent dès lors se munir d'outils facilitant la sélection et la classification de cette abondance de documents. Ces outils sont d'autant plus nécessaires quand on considère la variété de mentefacts

34 Musée de la personne. Musée de la personne: 
pouvant être intégrés au musée. Plusieurs typologies existantes permettent d'étudier les manifestations relevant du patrimoine immatériel. L'une des plus connues est celle de l'Organisation de Nations Unies pour l'éducation, la science et la culture (UNESCO) et révèle cinq domaines de pratiques: les traditions et les expressions orales; les arts du spectacle; les pratiques sociales, les rituels et les événements festifs; les connaissances et les pratiques concernant la nature et l'univers; et enfin les savoir-faire liés à l'artisanat traditionnel ${ }^{35}$. Ces domaines permettent l'identification et le classement des manifestations inscrites sur les Listes du patrimoine immatériel de l'UNESCO et font autorité en matière de patrimoine à l'échelle internationale. Parallèlement, la Grille des pratiques culturelles élaborée par Jean Du Berger constitue un travail fondamental dans le domaine de la classification du patrimoine ethnologique. Cette grille prend en considération l'ensemble des pratiques culturelles, traduites par des performances présentes ou passées, et est articulée autour de trois pôles: le champ coutumier, le champ pragmatique et le champ symbolique et expressif ${ }^{36}$. Ces trois champs, eux-mêmes divisés en neuf groupes de pratiques, tentent de représenter la complexité et le dynamisme des pratiques culturelles ${ }^{37}$.

En s'inspirant de ces deux typologies, il nous est possible d'adopter une position intermédiaire permettant de regrouper les différentes catégories de mentefacts s'intégrant au musée. Cette typologie se présente sous les cinq regroupements suivants: les pratiques langagières et orales; les expressions artistiques et les arts du spectacle; les pratiques rituelles, festives et coutumières; les savoir-faire techniques et traditionnels; les pratiques liées à l'individu et à son univers. En outre, le mentefact pourrait se trouver dans plus d'un ensemble à la fois,

35 UNESCO. Convention pour la sauvegarde du patrimoine culturel immatériel. <http://unesdoc. unesco.org/images/0013/001325/132540f.pdf> (consulté le 27 janvier 2010).

36 DU BERGER, Jean. Grille des pratiques culturelles. Québec: Septentrion, 1997,p. 23. en raison de son contenu qui peut être associé à plusieurs champs de pratique. Toutefois, son contenu demeure figé puisqu'il est inscrit sur un support; le mentefact ne peut donc changer d'ensemble d'une utilisation à l'autre.

L'étude s'est attardée au premier type, soit celui des pratiques langagières et orales, par le témoignage. Néanmoins, le témoignage oral, étant lui-même un type de patrimoine immatériel, peut rendre compte de pratiques liées aux autres catégories proposées. Par ailleurs, il est important de mentionner que ces mentefacts peuvent être présentés sous trois formes principales au musée: audio (seulement le son), vidéo (seulement l'image mouvante) et audiovidéo (le son et l'image mouvante). Afin de permettre l'intégration adéquate et optimale du mentefact au sein de leurs collections, il est essentiel que les institutions muséales se dotent d'un outil qui facilite leur sélection et justifie leur acquisition.

\section{Pour une grille de sélection du mentefact}

Favoriser une collecte ouverte et sans restriction permet indéniablement d'intégrer une quantité notable de sources orales au sein des activités du musée. Néanmoins, comme les études de cas analysées ont permis de le démontrer, cet engouement pour le mentefacttémoignage pourrait éventuellement causer problème en raison de l'ampleur que pourrait prendre un tel projet. Il est donc nécessaire de s'appuyer, tout comme dans le cas des artefacts, sur une grille de sélection des mentefacts permettant une collecte raisonnée.

37 Ces groupes, selon DU BERGER (ibid.), sont les suivants: pratiques coutumières, pratiques du corps, pratiques alimentaires, pratiques vestimentaires, pratiques techniques, pratiques ludiques et esthétiques, pratiques linguistiques, pratiques ethno-scientifiques et enfin pratiques éthiques. 
S’inspirant à la fois de la grille de valeurs proposée par Luc Noppen et Lucie K. Morisset concernant le patrimoine bâti et des critères d'acquisition mis de l'avant par Bibliothèque et Archives nationales du Québec, nous proposons ici une grille de critères adaptée aux particularités du mentefact ${ }^{38}$. Cette grille de sélection à deux niveaux permet d'évaluer le mentefact par rapport à l'institution dans un premier temps, et le mentefact en lui-même dans un second temps.

Les critères relatifs à l'institution énoncés ici permettent d'évaluer la pertinence du document par rapport au mandat de l'institution et la complémentarité du mentefact par rapport à d'autres documents (qu'ils soient déjà acquis ou en voie d'acquisition). Il s'agit aussi de déterminer si le document permet de répondre à des besoins de recherche ou de diffusion et de vérifier la disponibilité des moyens techniques pour conserver et diffuser le document, voire le transférer sur un nouveau support de conservation. Par ailleurs, les critères relatifs au mentefact visent l'évaluation du document en lui-même et de ses qualités intrinsèques. Ces critères concernent la valeur potentielle d'exposition, l'état de conservation du document, la qualité visuelle et sonore du document, l'absence de restriction à l'utilisation du document, sa rareté ainsi que le mode d'acquisition proposé. Il y a en outre d'autres critères de sélection qui s'attardent aux aspects inhérents à la nature singulière du mentefact, tributaire des avancées technologiques des dernières décennies. Tel est le cas de l'évaluation de la disponibilité de moyens techniques pour la conservation du document et de la qualité visuelle et sonore du mentefact. En somme,

38 La grille élaborée par Luc NOPPEN et Lucie K. MORISSET ( Ville et mort du patrimoine». In. DELORME, Pierre (dir.). La ville autrement. Québec: Presses de l'Université du Québec, 2005, p. 49-66) permet d'illustrer le processus de patrimonialisation de l'objet à l'aide des valeurs qu'on lui attribue. D'une part, les valeurs d'existence intègrent les valeurs d'âge, d'art, de position et de matérialité. D'autre part, la valeur d'usage permet d'évaluer la fonction (s'il s'agit d'un document) ou l'utilité (s'il s'agit d'un monument). Les critères d'acquisition établis par BIBLIOTHÈQUE ET ARCHIVES NATIONALES DU QUÉBEC (Politique d'acquisition des archives privées de BAnQ. $<$ http://www.banq.qc.ca/a_propos_banq/lois_reglements_ politiques/politiques_procedures/politique_acquisition_ cette grille, sans être trop restrictive, permet d'évaluer la pertinence d'un mentefact dans le contexte de sa possible intégration au musée. Une fois intégré aux collections, le mentefact peut être mis à contribution pour d'éventuels projets de mise en valeur, tels que les expositions. Dès lors, le mentefact entretient nécessairement une relation avec les autres objets et documents qui partagent son contexte de diffusion.

\section{Le mentefact et sa relation aux autres expôts}

Le mentefact peut être introduit de diverses manières à l'exposition muséale, comme en fait foi la présentation des réalisations du Musée de la mémoire vivante et du Centre d'histoire de Montréal. L'exposition regroupe des expôts de supports et de natures variés: artefacts, œuvres d'art, documents iconographiques, archives textuelles, etc. Quelle place le mentefact occupe-t-il ? Et surtout quel rapport entretient-il avec les autres objets et documents présentés? Le mentefact et les autres musealia peuvent être mis en relation selon trois aspects: dépendance, indépendance et interdépendance. D'abord, le mentefact peut être considéré comme dépendant d'un ou de plusieurs autres expôts. Il est donc intimement lié à cet(ces) autre(s) objet(s) par son contenu. Le mentefact, en raison de l'information qu'il diffuse, se trouve donc directement en lien avec l'objet exposé, puisqu'il y fait référence. Grâce à ce type de relation, l'objet bénéficie d'une riche valeur documentaire, puisque le propriétaire - actuel ou ancien permet de retracer la vie, ou du moins une partie de la vie, de l'objet. Ce mentefact ne

archives_privees/index.html> [consulté le 15 avril 2010]) traduisent pour leur part le processus d'intégration des archives dans les collections nationales. Dans ce contexte, BAnQ conçoit deux catégories de critères dans l'acquisition de documents: fondamentaux et secondaires. Les critères fondamentaux concernent: la valeur secondaire significative (fonction d'information), la complémentarité des documents avec les fonds d'archives existants et les besoins de la recherche. Les critères secondaires se rapportent à: l'originalité et l'authenticité des documents, la rareté, l'état des documents, le mode d'acquisition proposé, les conditions d'accès et d'utilisation, la valeur potentielle d'exposition et enfin l'authenticité et la propriété bien établies. 
peut donc pas, ou difficilement, être présenté sans l'objet auquel il est consacré. Ensuite, le mentefact peut également être inséré dans un contexte où il sera complètement indépendant des autres expôts. Dans ce cas, le mentefact est présenté pour son contenu, sans lien direct avec les autres objets et documents. Il a alors été recueilli pour ce qu'il représentait lui-même et non pour bonifier la valeur documentaire d'un objet. Par conséquent, il peut être présenté dans une diversité de contextes d'exposition, puisque son interprétation peut être multiple. Finalement, le mentefact peut entretenir un rapport d'interdépendance avec les autres expôts. Il se trouve alors dans une position intermédiaire entre les deux catégories précédentes. Sans être totalement lié à l'objet, il permet d'approfondir la connaissance de ce dernier. Dans ce cas, le mentefact est en lien avec l'autre expôt, mais pourrait tout aussi bien être présenté sans lui. Tout comme l'autre expôt, quoique bénéficiant de l'apport du mentefact, il pourrait être mis en valeur indépendamment de celui-ci. En outre, le mentefact et l'objet ou le document auquel il est associé s'enrichissement mutuellement.

\section{En conclusion: les limites du concept de mentefact}

Il est maintenant entendu que le mentefact s'intègre de manière cohérente dans le courant de dématérialisation de l'objet muséal et de virtualisation progressive du musée ${ }^{39}$. La reconnaissance du récit singulier face à l'histoire officielle a sans contredit influencé le musée dans l'intégration de la communauté au sein de ses activités. Dans cette même vague, le musée d'histoire et de société s'est empressé d'intégrer le patrimoine immatériel à ses activités par le biais du mentefact, véritable substitut et re-création d'un événement. Néanmoins, certaines limites à son intégration au musée, et plus particulièrement à l'exposition, sont à souligner.

39 DELOCHE, Bernard. Le musée virtuel: vers une éthique des nouvelles images. Paris: Presses universitaires de France, 2001. 40 MAIROT, «Musées et société», op. cit., p. 346.
D’abord, comme Philippe Mairot l'évoque, «au regard des recherches récentes et en cours, les musées n'apparaissent plus comme les discrets analystes des identités mais comme un de leurs facteurs $»^{40}$. Les intervenants du musée doivent donc être conscients de ce rôle fondamental qu'ils jouent dans le développement et l'appropriation d'une identité collective et agir avec prudence lorsqu'il s'agit de faire appel à la communauté. En effet, le mentefact doit permettre de traduire la diversité des opinions et refléter la société dans l'ensemble de sa complexité. Pour ce faire, il est important que, dès le stade de collecte, cette diversité soit prise en considération.

Par ailleurs, Serge Chaumier mentionne que «le musée de société ne doit pas se résumer à être une mise en ordre du passé, mais devrait se donner pour objectif de parler du présent. Les objets du passé n'ont de sens que s'ils s'ouvrent sur l'actuel et le quotidien ${ }^{41}$. De la même manière, le mentefact ne doit pas être recueilli et mis en valeur uniquement pour représenter certains aspects du passé, car cela serait restreindre son utilité à la représentation de certains thèmes et sujets pour lesquels aucun témoin matériel n'a été sauvegardé. Au contraire, il doit dépasser ce stade d'urgence de la sauvegarde et être orienté vers le présent et l'avenir. Dans cette perspective, le mentefact pourrait être l'objet permettant l'intégration efficace de l'actualité au musée. Le mentefact doit en outre être en mesure de révéler le changement, le mouvement, le dynamisme inhérent à toute société. En ce sens, les cliniques de mémoire réalisées par le Centre d'histoire de Montréal et le Musée de la personne de Montréal constituent des exemples de pratiques ancrées dans le présent, non sans faire référence à un certain passé. Le mentefact semble donc constituer l'outil approprié pour traiter du passé au présent, tout en permettant cette ouverture vitale vers l'avenir.

41 CHAUMIER, Serge. Des musées en quête d'identité: Écomusée versus technomusée. Paris: L'Harmattan, 2003, p. 124. 
Tableau 1

Typologie du mentefact proposée, s'inspirant à la fois

de la typologie du patrimoine immatériel de l'UNESCO

et de la Grille des pratiques culturelles de Jean Du Berger.

\begin{tabular}{|l|l|}
\hline $\begin{array}{l}\text { Les pratiques langagières } \\
\text { et orales }\end{array}$ & $\begin{array}{l}\text { Permet de traduire un vaste champ de } \\
\text { manifestations, telles que: les expressions } \\
\text { orales, les récits de vie, les témoignages, } \\
\text { la littérature orale, les contes et légendes, } \\
\text { les récits d'expériences, les blagues, pour } \\
\text { ne nommer que celles-ci. }\end{array}$ \\
\hline $\begin{array}{l}\text { Les expressions artistiques } \\
\text { et arts du spectacle }\end{array}$ & $\begin{array}{l}\text { Ce champ regroupe les pratiques liées à } \\
\text { la danse, au chant, à la musique instrumenta- } \\
\text { les et vocale, au théâtre, au cinéma, etc. }\end{array}$ \\
\hline $\begin{array}{l}\text { Les pratiques rituelles, } \\
\text { festives et coutumières }\end{array}$ & $\begin{array}{l}\text { Ce vaste ensemble comprend, entre autres: } \\
\text { les pratiques liées aux croyances religieuses, } \\
\text { les commémorations, les fêtes, les rites } \\
\text { de passage, etc. }\end{array}$ \\
\hline $\begin{array}{l}\text { Les savoir-faire techniques } \\
\text { et traditionnels }\end{array}$ & $\begin{array}{l}\text { Ces savoir-faire peuvent être liés aux arts } \\
\text { et métiers, aux pratiques de production } \\
\text { (l'agriculture, par exemple), aux pratiques } \\
\text { sportives, etc. }\end{array}$ \\
\hline $\begin{array}{l}\text { Les pratiques liées à } \\
\text { individu et son univers }\end{array}$ & $\begin{array}{l}\text { Cet ensemble regroupe d'une première part, } \\
\text { les pratiques liées au corps, à l'alimentation } \\
\text { et à l'habillement et, d'une seconde part, } \\
\text { les pratiques liées à la relation de l'individu } \\
\text { à son univers (les pratiques ethno-scientifiques } \\
\text { selon Du Berge: flore, faune, métérologie, } \\
\text { etc.). }\end{array}$ \\
\hline
\end{tabular}




\section{Tableau 2}

Grille de sélection du mentefact proposée, s'inspirant à la fois de la grille de valeurs proposée par Luc Noppen et Lucie K. Morisset concernant le patrimoine bâti et des critères d'acquisition mis de l'avant par Bibliothèque et Archives nationales du Québec.

\begin{tabular}{|l|}
\hline Critères relatifs à l'institution \\
Pertinence par rapport au mandat \\
de l'institution \\
Complémentarité par rapport \\
à d'autres documents (déjà acquis \\
ou en voie d'acquisition) \\
Permet de répondre à des besoins \\
de recherche et/ou de diffusion \\
Disponibilité des moyens techniques \\
pour conserver et diffuser le document
\end{tabular}

Critères relatifs au mentefact
Valeur potentielle d'exposition
État de conservation du document
Qualité visuelle ou sonore du document
Absence de restriction à l'utilisation
du document
Rareté
Mode d'acquisition proposé


Par ailleurs, il est indéniable que l'urbanisation et la mondialisation permettent, plus que jamais, le mouvement des individus. Le mentefact ne doit donc plus être seulement garant d'une mémoire d'un passé collectivement partagé, mais doit représenter une mémoire du changement.

[L]e phénomène d'urbanisation devient l'argument latent d'une mémoire collective du changement. Certes cette mémoire collective du changement ne s'enracine pas dans des groupes sociaux, des espaces ou des temps pré-existants, mais elle existe et elle est le germe potentiel de nouveaux groupes comme de nouveaux systèmes spatio-temporels. Et c'est en cela que réside le changement de mémoire collective ${ }^{42}$.

En somme, le mentefact, de nature dynamique, doit également s'intéresser à ce mouvement social. Dans un tout autre ordre d'idées, bien que de plus en plus accessibles, les nouvelles technologiques ne sont pas à la portée de toutes les institutions muséales, en raison des coûts qu'elles entraînent. Ainsi, la diffusion du mentefact, puisque intimement liée à la technologie, n'est pas accessible à tous les musées, notamment ceux de petite taille. Néanmoins, l'accès globalement étendu du réseau Internet permet aux institutions de proposer des ressources virtuelles, comme le fait l'ensemble des Musées de la personne. La virtualisation du musée permet indéniablement une diffusion importante du mentefact. Que ce soit par des expositions virtuelles ou par l'entremise de leur site Internet, les institutions muséales adhèrent de plus en plus à ce type de diffusion. Si le musée tend à sa dématérialisation et à sa virtualisation, l'individu n'y est pas pour autant évincé. Sa contribution y est au contraire de plus en plus requise. Comme Mathieu Dormaels le rappelle, «mais où sont ceux qui font le musée? Parce que même si chacun peut laisser son histoire ou sa mémoire, en ligne ou ailleurs, on ne fait là que constituer

42 DAGHINI, Giairo, Pascal AMPHOUX, Michel BASSAND, André DUCRET, Dominique JOYE, Yves PEDRAZZINI et Nicole VALIQUER. Mémoire collective et urbanisation. Genève: Centre de recherche sur le phénomène urbain et Institut de recherche sur l'environnement construit, 1988, p. 201. une 'collection' et il est certain qu'il faudra toujours quelqu'un pour la préserver, faire des recherches et diffuser ces connaissances ${ }^{43}$." L'individu est donc présent au musée, il est à la fois responsable de son fonctionnement, sujet de son étude et, grâce au mentefact, il devient désormais objet d'exposition.
43 DORMAELS, Mathieu. "Rôle social des musées: une autre 'nouvelle' muséologie". Muséologies Les cahiers d'études supérieures, vol. 2, n 2, 2008, p. 121. 
Ariane Blanchet-Robitaille, translated by Allana Carlyle

\section{Mentefacts in Museums: Staging Memory}

For the last few decades, the museum has been confronted with an increasing preoccupation for the individual and their intangible products. The new museology movement and the practices which it has inspired have well demonstrated that the material object is no longer sufficient in itself for the complex representation of society and its history. In this context, history and society museums have invested in new research and practices and have progressively integrated new intangible objects into the heart of their work. These new objects, musealia as a whole, will be defined here as the term mentefact, the intangible products of Man. Along with this overture to the field of intangibility, major technological progress has allowed the museal institution to renew its museography and open it to new possibilities. Finally, the recognition of individual experience throughout contemporary history, justifies the museum's keen interest in oral testimony over the last few decades. This context of emergence has allowed the mentefact to define itself as a museal object, a substitute and re-creation of a unique past event.

Different understandings underlie the concept of the mentefact, depending on the author and the context. Whether it is the definition set out by the department of Culture, des Communications et de la Condition feminine, where mentefact is defined as "the ensemble of spiritual or intangible productions which constitute the intangible section of ethnological heritage " " or the definition of Jan Dolák which considers the museal collection as follows: "it can be an artefact (created by Man), a "naturfact» (created by nature) or a "mentefact" (a recording of the intangible manifestations of man), subject in all cases to strict selection criteria ${ }^{2}$, it is evident that the desire to differentiate material from immaterial productions leads the reflection on museal objects. In addition, it appears that the transition from "un-recorded mentefacts", such as Jean Du Berger conceives cultural practices, to mentefacts as "museum objects", is intimately linked to the technical progress of the last centuries. The mentefact therefore constitutes the witness of what was produced, a re-creation, a substitute of the event without being the event itself. It requires support material (magnetic tape, CD, DVD, etc.)

1 Ministère de la culture, des Communications et de la Condition féminine. (2010, January 30). In Gouvernement du Québec, Glossaire. Retrieved January 30, 2010 from http://www.mcccf.gouv.qc.ca/index.php?id=1899 This definition is also shared by the Politique du patrimoine de la République française.

2 Dolák, Jan. (2008). Museology as a tool for a modern museum. Actes du colloque Tourisme culturel: tendances et strategies. Montreal, ICOM, Pointe-à-Callière, Montreal Museum of Archaeology and History, Montreal Science Centre. Retrieved March 27, 2010 from http://www.montrealceca2008.org/12.html

3 Du Berger, J., Turgeon, L., Létourneau, J. \& Fall, K. (1997). Prolégomènes à une ethnologie urbaine. Les espaces de l'identité. Sainte-Foy : Presses de l'Université de Laval. 
in order to ensure its conservation and above all its diffusion, while still being considered independently of the latter. Oral testimony constitutes one of the many types of mentefacts that the museum integrates into its work. In Quebec, certain institutions such as the Musée de la mémoire vivante in Saint-Jean-Port-Joli and the Musée de la personne in Montreal gear their actions towards testimonial-mentefacts.

In the context of an increasing consideration for intangible heritage, is it now essential that museal institutions acquire the necessary tools to allow for the documentation and acquisition of mentefacts. It would therefore also be pertinent to implement a typology, following the examples of both UNESCO for intangible heritage and the Grille des pratiques culturelles of Du Berger and being able to articulate according to the following categories: linguistic and oral practices; artistic expressions and performing arts; ritual, festival and customary practices; technical and traditional know-how; practices related to the individual and his/her universe. In this same line, a selection grid allows for the evaluation of the relevance of mentefacts, similar to the criteria grid in use for the acquisition of objects, and will be necessary to facilitate the integration of the mentefact into the museal collection. This grid could consider both the criteria concerning the institution (relevance of the mentefact in relation to the mandate of the institution, its compliment to other documents acquired or in the process of being acquired, and whether it responds to research needs and/or the diffusion and availability of technical means to conserve and diffuse the document) and criteria relative to the mentefact itself (potential exhibition value, state of conservation of the document, visual and sonorous quality of the document, absence of restrictions relating to use of the document). These tools confirm the recognition of the mentefact as a museal object as a whole and will facilitate its integration into the museum.

It is now possible to accept that the mentefact has consistently integrated into the movement of the dematerialisation of the object and the virtualisation of the museum. In addition, the emergence of this object of intangible substrate allows us to see that although the museum is moving towards a permanent virtualisation, the individual has not been unseated. On the contrary, the individual's contribution is increasingly needed as it is responsible for both its own functioning, is the subject of its own study and thanks to the mentefact, is also an exhibition object. 